used for quantitative real-time PCR, with multiparameter flow cytometry utilised to measure hydrogen peroxide levels, apoptosis, DNA damage and cell proliferation.

Results DUOX2 is expressed throughout the colonic epithelium, is upregulated in active compared to quiescent ulcerative colitis and also in areas of UC associated dysplasia. In the setting of intestinal inflammation, but not in quiescent disease, 5-ASA enhances DUOX2 expression in vivo and ex vivo. As expected, 5-ASA was found to suppress cytokine (IL-6 and IL-8) production during an inflammatory flare and to maintain low cytokine levels during remission. The addition of 5-ASA in vitro led to upregulation of DUOX2 and elevated levels of hydrogen peroxide, DNA damage and apoptosis. These effects were further enhanced in a setting of hypoxia.

Conclusion We have shown that 5-ASA over stimulates DUOX2 expression in the setting of inflammation and hypoxia, but not in quiescent disease. Importantly, this suggests that during a flare 5ASA could act as a carcinogen rather than a chemo-preventative drug. Further investigations to confirm the functional relevance of DUOX2 up-regulation in the colonic mucosa of patients with active UC is indicated.

Competing interests T Macfie Grant/Research Support from: SHIRE, A Parker: None declared, A Nijhuis: None declared, T Boitsova: None declared, N Suraweera: None declared, R Jeffery: None declared, R Poulsom: None declared, J Lindsay Grant/Research Support from: SHIRE, A Silver: None declared.

\section{PWE-256 INTESTINAL INFLAMMATION REGULATES RETINOIC ACID DEPENDENT IMPRINTING OF GUT TROPISM BY DENDRITIC CELLS INDEPENDENTLY OF RALDH EXPRESSION}

doi:10.1136/gutjnl-2012-302514d.256

${ }^{1} \mathrm{~T}$ J Sanders, ${ }^{*}{ }^{1} \mathrm{~N}$ E McCarthy, ${ }^{1} \mathrm{E}$ Giles, ${ }^{2} \mathrm{~J} 0$ Lindsay, ${ }^{1} \mathrm{~A}$ J Stagg. ${ }^{1}$ Centre for Immunology and Infectious Disease, Blizard Institute, London, UK; ${ }^{2}$ Digestive Diseases Clinical Academic Unit, Barts \& The London School of Medicine \& Dentistry, London, UK

Introduction In the mouse, tissue-specific expression of retinaldehyde dehydrogenase (RALDH) enzymes by CD103+ intestinal dendritic cells (DC) enables them to generate all-trans retinoic acid (RA) and thereby imprint a gut-tropic phenotype on T cells via induction of homing receptors including $\alpha 4 \beta 7$ integrin. In health, RA from $\mathrm{CD} 103+$ also enhances their generation of Treg, contributing to intestinal homeostasis. In murine models of inflammatory bowel disease (IBD) RALDH expression by CD103+ DC is reduced but little is known about the function of RA in the human intestine. The aim of this study was to determine whether factors present in the healthy and inflamed human intestine regulate RA generation and activity.

Methods Conditioned media (CM) were generated by culture of intestinal biopsies from healthy individuals and IBD patients (inflamed/non-inflamed regions). DC were differentiated from monocytes using GM-CSF and IL-4 in the presence or absence of CM. Expression of RA-generating enzymes was assessed by qRTPCR and RALDH activity determined using the Aldefluor assay. Induction of $\alpha 4 \beta 7$ following activation of naive allogeneic CD4+ T cells was determined by flow cytometry.

Results Activation of naive CD4+ T cells by human monocytederived DC resulted in RA-dependent upregulation of $\alpha 4 \beta 7$. These DC possessed retinal-inhibitable Aldefluor activity and expressed both alcohol dehydrogenase $(R D H 10)$ and RALDH $(R A L D H 1,2,3)$ enzymes required for the generation of RA from retinol via retinal. Aldefluor activity was regulated by GM-CSF and RA, and reflected predominately the activity of RALDH2 as suggested by qRT-PCR analysis of sorted Aldefluor+ DC. CM significantly suppressed Aldefluor activity $(p<0.0001)$ irrespective of whether generated from healthy or IBD tissue (inflamed or non-inflamed). The inhib- itory effect of CM generated from healthy tissue could be partially reversed with the prostaglandin E2 (PGE2) EP-2 receptor antagonist AH6809 but this effect was less marked with CM from IBD tissue suggesting the involvement of distinct RALDH regulators. Although the effects of inflamed and non-inflamed CM on Aldefluor activity were similar, DC differentiated in the presence of inflamed $C M$ induced significantly higher $(\mathrm{p}<0.05)$ levels of CD4 T cell $\alpha 4 \beta 7$ expression.

Conclusion Factors generated in the human intestinal mucosa limit RALDH activity in DC and may thereby impact upon their generation of RA. Factors other than PGE2 are involved particularly in inflamed tissue. Intestinal mediators influence the imprinting of gut tropism independently of effects on RA-generating enzymes. Manipulation of RA availability may offer new therapeutic options in IBD.

Competing interests None declared.

\section{PWE-257 THE ROLE OF RDH10 AND RALDH ENZYMES IN RETINOIC ACID-MEDIATED IMMUNE REGULATION BY ANTIGEN PRESENTING CELLS IN THE HUMAN INTESTINE}

doi:10.1136/gutjnl-2012-302514d.257

${ }^{1} \mathrm{~T}$ J Sanders, ${ }^{*} \mathrm{~N}$ E McCarthy, ${ }^{1} \mathrm{E}$ M Giles, ${ }^{2} \mathrm{~J} 0$ Lindsay, ${ }^{1} \mathrm{~A} J$ Stagg. ${ }^{1}$ Centre for Immunology and Infectious Disease, Blizard Institute, London, UK; ${ }^{2}$ Digestive Diseases Clinical Academic Unit, Barts \& The London School of Medicine \& Dentistry, London, UK

Introduction All-trans retinoic acid (RA) has emerged as an important immunoregulatory molecule with specific functions in the intestine. RA is generated enzymatically by the sequential action of alcohol dehydrogenase $(\mathrm{ADH})$ and retinaldehyde dehydrogenase (RALDH) enzymes. In the mouse, RALDH expression is confined to $\mathrm{CD} 103+$ intestinal dendritic cells (DC) giving this subset the unique ability to mediate RA-dependent functions such as the imprinting of gut tropism on T cells. RALDH activity is downregulated in mouse models of inflammatory bowel disease (IBD) but little is known about its role in human intestine. The aim of this study was to assess RA-dependent immune regulation by human intestinal antigen presenting cells (APC).

Methods Lamina propria mononuclear cells (LPMC) were extracted from intestinal biopsies by enzymatic digestion and analysed by multicolour flow cytometry. Purified subsets of intestinal APC were obtained by immunomagnetic or FACS sorting. Expression of $A D H$ and RALDH enzymes was quantified by quantitative RT-PCR and RALDH activity assessed using the Aldefluor assay. The ability to induce the gut homing receptor $\alpha 4 \beta 7$ on T cells was assessed by flow cytometry following stimulation of CFSE-labelled naïve CD4+ T cells.

Results Induction of the gut homing receptor $\alpha 4 \beta 7$ on human T cells was RA dependent. Intestinal myeloid APC were potent stimulators of naive CD4+ T cells and induced high levels of $\alpha 4 \beta 7$. Both of these activities were attributed to the CD103+ APC fraction. However, analysis of ex vivo intestinal populations revealed RALDH activity in all myeloid APC populations studied: CD103+ and CD103- DC identified as HLA-DR+CD11c+Lin- (Lin=anti-CD3,14,16,19,34) as well as HLA-DR+CD11c+Lin+ monocyte-like cells. Lymphocytes had little or no Aldefluor activity. Comparison of intestinal DC from healthy controls and IBD patients showed similar RALDH activity in inflamed and non-inflamed tissue. RALDH activity was equivalent in $\mathrm{CD} 103+$ and $\mathrm{CD} 103-\mathrm{DC}$. In contrast, the $\mathrm{ADH}$ RDH10 was expressed at levels 4.5-fold higher in CD103+ DC.

Conclusion As in the mouse, RA-mediated induction of $\alpha 4 \beta 7$ is a property of the CD103+ subset of intestinal DC. However in divergence from murine data, this property is associated with restricted expression of the $\mathrm{ADH}$ enzyme $\mathrm{RDH} 10$ rather than 
"downstream" RALDH enzymes. These data imply that RA availability is regulated differently in mice and man, with expression of $\mathrm{RDH} 10$ providing an important control point in humans.

Competing interests None declared.

\section{PWE-258 THIOPURINE MONITORING IN INFLAMMATORY BOWEL DISEASE PATIENTS AT A DISTRICT GENERAL HOSPITAL}

doi:10.1136/gutjnl-2012-302514d.258

${ }^{1} \mathrm{~T}$ Jowitt, ${ }^{*} \mathrm{C}$ Bowman, ${ }^{2} \mathrm{M}$ Ahmed, ${ }^{2} \mathrm{~S}$ Singh, ${ }^{2} \mathrm{C} \mathrm{H}$ Lim. ${ }^{1}$ Medical School, University of Birmingham, Birmingham, UK; ${ }^{2}$ Department of Gastroenterology, Good Hope Hospital, Sutton Coldfield, UK

Introduction Thiopurines are an unlicensed but recognised therapy for Inflammatory Bowel Disease (IBD). These drugs interfere with cell signalling and have significant side effects including leucopaenia, pancreatitis and hepatotoxicity. Therefore routine monitoring of blood is mandatory. Our Trust has guidelines (based on BSG guidelines) for the monitoring of Thiopurine therapy. Patients are monitored by a IBD Nurse specialist using a simple electronic database.

Methods Objective: To determine the effectiveness of the IBD Nurse database in ensuring that patients on Thiopurines are monitored according to Trust guidelines. A total of 900 patients with IBD attend our gastroenterology clinics. Of these 204 are on Thiopurines. Trust guidelines recommends weekly blood test monitoring for the first month starting Thiopurines. This is followed by monthly for the next 3 months and every 3 months subsequently. Patients latest blood results were collected over a two consecutive days in July 2011 for 204 patients. Patients more than 7 days late for blood tests were considered non-compliance with the guideline.

Results 182 (89\%) patients were having their bloods monitored as per trust guidelines. However 22 patients (11\%) were being monitored incorrectly, with a median of 68.5 (32.25-269.25) days overdue. 16 of these patients have a diagnosis of Crohn's Disease, the remaining six have Ulcerative Colitis. 14 (7\%) patients had either deranged liver function tests or were neutropaenic.

Conclusion The current manual database is reliable as the majority of thiopurines patients were being monitored as per hospital guideline. However, $7 \%$ of abnormal blood results may not be recognised promptly and no action was taken. An automated database with automated reminder to patients who passed their blood test due date and electronic notice to the doctor responsible for the patient is needed to reduce the potential risk of harm to the patients.

Competing interests None declared.

\section{REFERENCE}

1. Anon. Guidelines for the management of inflammatory bowel disease in adults. Gut 2011;60:571-607.

\section{PWE-259 THE EFFICACY OF METHOTREXATE IN CROHN'S DISEASE: A CLINICAL PERSPECTIVE}

doi:10.1136/gutjnl-2012-302514d.259

T T Gordon-Walker,* M W Stahl, M Groome, J Todd, N Reynolds, C Mowat. Department of Gastroenterology, Ninewells Hospital, Dundee, UK

Introduction Methotrexate (MTX) has been shown in clinical trials to be effective in the induction and maintenance of remission in Crohn's Disease (CD). It is predominantly used in patients intolerant of, or whose disease is refractory to, thiopurine treatment. It was our aim to examine, in a clinical setting, the efficacy and sideeffect profile of MTX in patients with CD.
Methods A retrospective audit was performed by casenote review of all patients with $\mathrm{CD}$ attending the clinical investigations unit at Ninewells Hospital to commence parenteral MTX during the period 1 January 2007 to 31 December 2010. A total of 53 patients were identified, for whom casenotes were available in 52 (26 male/26 female). Intramuscular MTX treatment was initiated at a dose of $25 \mathrm{mg}$ once weekly for 16 weeks, followed by oral MTX at a dose of $15 \mathrm{mg}$ once weekly. Clinical response by physician's global assessment was recorded at 3, 6 and 12 months. Clinical response was defined as complete response (absence of IBD symptoms or complete healing of fistulae); partial response (symptomatic improvement but ongoing symptoms); or no response (no improvement or deterioration from baseline). Relapse was defined as deterioration in symptoms necessitating additional medical or surgical intervention.

Results Median age of starting MTX was 32 (range 15-73). 51 of 52 patients had previously received thiopurine treatment. 12-month follow-up data were available for 48 patients. 34 patients were taking steroids at the time of initiating MTX. At 12 months, complete response was reported in 8 patients $(17 \%)$, partial response in 6 patients $(13 \%)$, no response in 21 patients $(44 \%)$ and drug withdrawal due to side effects in 13 patients (27\%). Relapse was reported in 17 patients (35\%). 10 patients (21\%) required additional medical therapy (steroids or biological therapy). Surgical intervention was required in seven patients (15\%). Steroid withdrawal at 1 -year, without recourse to biological or surgical therapy, was reported in only 11 of 34 patients $(32 \%)$. MTX-associated side effects were reported in 25 patients (48\%). Reported side effects included: LFT abnormalities (9), nausea (8), lymphopenia (5), lethargy (3) and mouth ulceration (1). Monitoring of FBC/LFTs was performed at $97 \%$ of scheduled weekly intervals for patients receiving parenteral MTX and at $74 \%$ of scheduled monthly intervals for patients on oral MTX.

Conclusion In this cohort, the clinical effectiveness of MTX in the induction and maintenance of remission of CD was limited. Only $29 \%$ of patients had either a complete or partial response to therapy at 1-year. The clinical effectiveness of MTX was limited by sideeffects in $27 \%$. On the basis of these results we should re-consider the position of MTX in the management of CD

Competing interests None declared.

\section{PWE-260 OPTIMAL C REACTIVE PROTEIN CUT-OFF POINT FOR PREDICTING HOSPITALISATION IN PATIENTS WITH MODERATELY ACTIVE CROHN'S DISEASE}

doi:10.1136/gutjnl-2012-302514d.260

${ }^{1} \mathrm{~J}$-F Colombel, ${ }^{2} \mathrm{~W}$ J Sandborn, ${ }^{3} \mathrm{E}$ Louis, ${ }^{4} \mathrm{R}$ Panaccione, ${ }^{5} \mathrm{R}$ B Thakkar, ${ }^{5} \mathrm{M}$ M Castillo, ${ }^{5} \mathrm{M}$ Yang, ${ }^{6} \mathrm{~T}$ Finney-Hayward, ${ }^{*}{ }^{5} \mathrm{~J}$ Chao, ${ }^{5} \mathrm{P} \mathrm{M}$ Mulani. ${ }^{1}$ Centre Hospitalier Universitaire de Lille, Lille, France; ${ }^{2}$ UCSD, La Jolla, California, USA; ${ }^{3} U$ of Liège, Liège, Belgium; ${ }^{4} U$ of Calgary, Calgary, Alberta, Canada; ${ }^{5}$ Abbott Laboratories, Abbott Park, Illinois, USA; ${ }^{6}$ Abbott Laboratories Ltd, Maidenhead, Berkshire, UK

Introduction To identify high risk patients among patients with moderate Crohn's disease (CD), we explored the association between $C$ reactive protein (CRP) concentration and hospitalisation risk for patients with moderately active CD and identified the optimal CRP cut-off point as a marker to predict CD-related hospitalisation. CRP is a well-studied and commonly used laboratory marker of inflammation in CD. ${ }^{1}$ The relationship between CRP and hospitalisation risk given the same Crohn's Disease Activity Impairment (CDAI) score in patients with moderate CD has not been studied.

Methods Data from CHARM, a 56-week (wk), randomised, placebocontrolled trial of adalimumab maintenance therapy, were analysed. All patients received adalimumab during a 4-wk, open-label induction period; patients were then randomised to adalimumab or placebo for a 52 -wk double-blind period. For this analysis, only patients who were randomised to placebo at Wk 4 and had 